\title{
A Workload-driven Document Database Schema Recommender (DBSR)
}

\author{
Vincent Reniers $(\bowtie)^{[0000-0003-3895-702 X]}$, Dimitri Van \\ Landuyt ${ }^{[0000-0001-6597-2271]}$, Ansar Rafique ${ }^{[0000-0002-5881-7588]}$, and Wouter \\ Joosen ${ }^{[0000-0002-7710-5092]}$ \\ imec-DistriNet, KU Leuven \\ Celestijnenlaan 200A, 3000 Leuven, Belgium \\ first.lastname@cs.kuleuven.be
}

\begin{abstract}
Database schema design requires careful consideration of the application's data model, workload, and target database technology to optimize for performance and data size. Traditional normalization schemes used in relational databases minimize data redundancy, whereas NoSQL document-oriented databases favor redundancy and optimize for horizontal scalability and performance.

Systematic NoSQL schema design involves multiple dimensions, and a database designer is in practice required to carefully consider (i) which data elements to copy and co-locate, (ii) which data elements to normalize, and (iii) how to encode data, while taking into account factors such as the workload and data model.

In this paper, we present a workload-driven document database schema recommender (DBSR), which takes a systematic, search-based approach in exploring the complex schema design space. The recommender takes as main inputs the application's data model and its read workload, and outputs (i) the suggested document schema (featuring secondary indexing), (ii) query plan recommendations, and (iii) a document utility matrix that encodes insights on their respective costs and relative utility. We evaluate recommended schema in MongoDB using YCSB, and show significant benefits to read query performance.
\end{abstract}

Keywords: NoSQL database design - Document database schema recommender $\cdot$ NoSQL database cost models

\section{Introduction}

In the Big Data era, the systematic collection of enormous data volumes has become the norm in various industry sectors, such as Healthcare, E-commerce and Telecom. Heterogeneous data emerges from many sources and in large volumes, from users, sensors, to logging mechanisms. These have to be stored and processed appropriately in database systems that can scale horizontally.

The un- or semi-structured nature of these data elements, coupled with their large influx, poses challenges to traditional relational database systems which 
adhere to a strict and rigid schema, and are limited in elastic and horizontal scalability. In order to handle such heterogeneous data, a new class of database systems has emerged, commonly referred to as NoSQL $[3,12,21]$. These provide support for specialized data models and features such as schema flexibility, leading to improvements in horizontal and elastic scalability. These benefits are achieved by relaxing the ACID properties of relational databases, in favor of eventual consistency [21], and secondly, by simplifying standard SQL operations [12].

General schema design guidelines for document-oriented NoSQL databases [10,14], such as MongoDB [2] and Couchbase [1], recommend data denormalization by embedding data elements (or copies thereof) into a single record (i.e. a document) to allow them to be served in a single look-up request. As table records are horizontally partitioned (sharded) across multiple nodes, a look-up may involve a costly JOIN operation traversing several physical nodes, which in turn significantly increases latency and overall strain on the database. Furthermore, some NoSQL databases do not support JOIN operations and favor denormalization, for example in MongoDB when using sharded collections, and instead these queries have to be implemented client-side. The main disadvantage of denormalization is that data redundancy will increase, and maintaining consistency across multiple copies will involve multiple write and update operations.

In general, the different options to denormalize NoSQL database schemas vary widely $[3,11,14]$, and each single schema design decision will in effect represent a trade-off between aspects of data locality, storage usage, consistency, performance, and scalability. Making appropriate trade-off decisions requires indepth knowledge of the application data model and its workload [19], the capabilities of the target database, and thus requires extensive expert knowledge. In practice, the database designer (or application developer) is fraught with finding a balance between normalization and de-normalization. In essence, this is a multi-dimensional optimization problem in which the combinatorial explosions of schema design possibilities hinders the ability to systematically enumerate this design space and search-based approaches are required [8].

In recent years, schema recommenders - mainly for columnar data storeshave emerged in related work (e.g., NoSE [18] or Bermbach et al. [5]) that implement such a search-based approach. These recommender systems take an (i) application's data model, and (ii) query workload, and suggest an appropriate NoSQL schema. However, to our knowledge, a systematic approach has not been presented for flexible NoSQL document-oriented databases.

In this paper, we propose DBSR, a document-based schema recommender that generates candidate schemas and employs a search-based approach to identify the most suitable candidates (evaluated in terms of query plan efficiency and projected cost). To evaluate DBSR, we have extended the Yahoo! Cloud Serving Benchmark (YCSB) [9] with dedicated workloads that integrate DBSR query plans. Our evaluation efforts show that significant benefits can be achieved in read query performance, but that this comes at an additional storage cost. 
The remainder of the paper is structured as follows, Section 2 motivates the paper and explains the used terminology. Section 3 introduces DBSR in terms of its algorithms and data structures. Section 4 elaborates on the pruning algorithms for query plans and documents. Section 5 evaluates DBSR, with emphasis on the pruning algorithms, and the generated recommendations. Finally, Section 6 discusses related work, whereas Section 7 concludes the paper.

\section{Motivating Example and Terminology}

The motivating example for this paper is presented in Figure 1. This example is based on an online auction system, and adopted from the RUBiS benchmark [6]. Starting from the ER model, a graph can be created with tables as nodes, and relationships as edges. At each point in the graph, a document can be constructed using the node as the top-level document, with options to either embed or reference the relationships to other nodes. The resulting document $D$ can be defined as a directed acyclic graph (DAG).
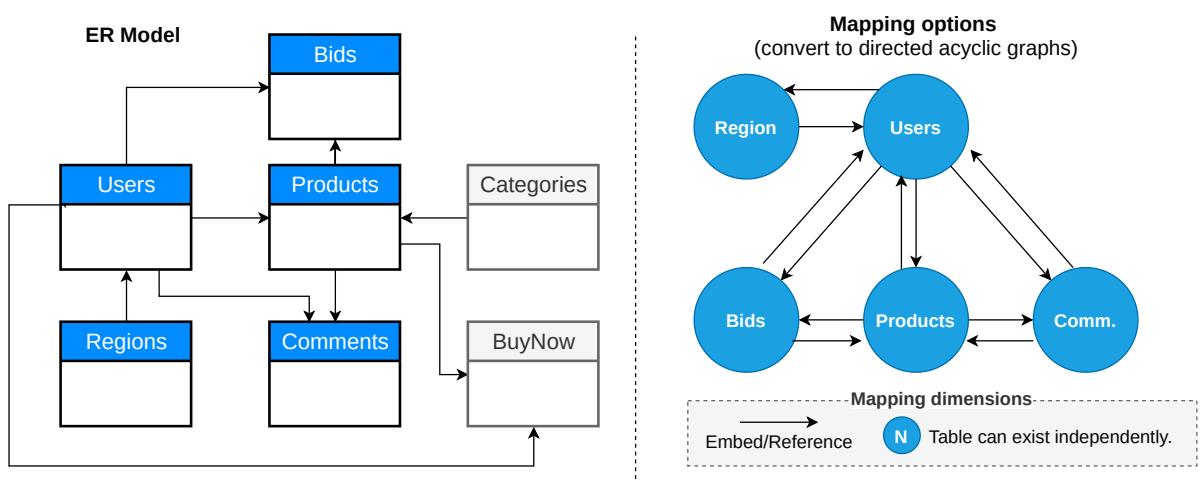

Fig. 1. RUBiS conceptual data model and mapping options.

It is a main rule of thumb in NoSQL design guidelines to group data that are frequently accessed together $[1,2]$. When a client visits a Product page, typically this person will be interested in the ongoing Bids for this Product, as well as in Comments made by previous buyers. Document collections optimized for the workload JOIN sequences $S e q_{P \rightarrow B}$ and $S e q_{P \rightarrow C}$ service this information with a single look-up without traversing documents $D_{B}$ containing a Bid or document $D_{C}$ containing a Comment separately, since these documents may be horizontally partitioned across several physical nodes. The optimized documents $D$ for each sequence are $D_{P \mid B}$ and $D_{P \mid C}$, of which $P \mid B$ and $P \mid C$ represent an embedded relationship, resulting in a single Product record that respectively embeds its Bids or Comments. The workload read sequence $S e q_{P \rightarrow B}$ its original query plan $Q P_{P \rightarrow B}$ is then replaced by a single look-up plan $Q P_{P \mid B}$. Further optimization 
creates document $D_{P \mid\{B, C\}}$ which embeds in a Product record both Bids and Comments. This example its document tree consists of a maximum height $(H)$ of 2 , and at each child a width $(W)$ of maximum 2 embedded relationships. An additional relationship $X$ embedded in this document can be clarified using brackets, for example by replacing $B$ with $\{B \mid X\}$, increasing overall height to 3 .

Although each query can potentially be optimized in this manner, this would quickly lead to significant increases in data redundancy, since each relationship copies the referenced data and embeds it locally.

Problem Statement. This example illustrates the complexity of the solution space, even for a simplified example. Designing a schema demands in-depth knowledge of the workload and its data model, and the capabilities of the target database, and involves making trade-offs between query performance, data redundancy, data consistency, etc.

Approach. In this paper, we adopt a search-based approach to generate candidate schemas and automatically rank them in terms of relative query plan efficiency and projected cost. Next, we explain how our schema recommender (DBSR) supports this process.

\section{Document-based Schema Recommender}

The DBSR framework ${ }^{1}$ architecture shown in Figure 2, is comprised of input models, a workload-driven generator, cost and ranking algorithms, a pruner, and output models.

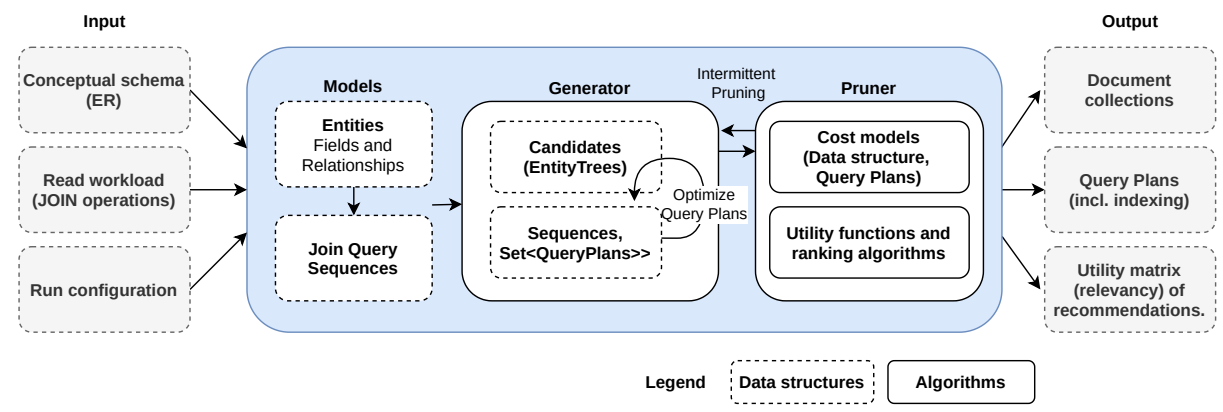

Fig. 2. DBSR: Architectural overview.

The generator takes as main inputs (i) the application's data model, (ii) a read workload of JOIN queries, and (iii) additional configuration parameters. The framework eventually outputs: (i) a document schema with secondary indexing, (ii) suggested query plans over these document collections, and (iii) a document utility matrix showing the relative efficiency in answering workload queries.

\footnotetext{
${ }^{1}$ DBSR framework repository: https://github.com/vreniers/DBSR
} 


\subsection{Workload-driven Schema Generation}

As discussed in [20], two alternative approaches can be adopted to generate document data structures from an input workload: either a top-down, or bottomup approach. We apply a bottom-up approach, which is globally more efficient, and starts from single-level document data structures for each table, rather than entirely denormalized documents (i.e. materialized views). Initial query plans are created from the workload that JOIN these single-level documents in multiple steps. Each query plan is optimized by merging used data structures into a larger document, and thereby eliminating a JOIN step that may traverse physical nodes.

Iterative Optimization of Query Plans. Figure 3 depicts the iterative loop of the generator. When a query plan is selected for optimization, the generator merges documents to eliminate a join step, thereby creating new larger documents, and a shortened query plan. All other query plans, that are notified of the novel data structure, can create new query plans based on the original plan and the new document. These plans are also added onto the stack for optimization.

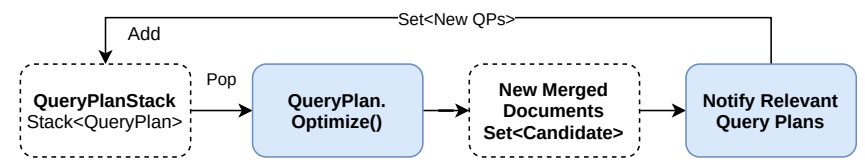

Fig. 3. Iterative loop optimizes query plans, which generates new documents and plans.

Stop Conditions. The generator will eventually stop when either (a) the query plan optimization stack becomes empty as no new data structures are generated and thus no novel query plans are added onto the stack, or (b) the maximum number of iterations (as stated in the run configuration) is reached.

\subsection{Initial Query Plans and Data Structures}

The iterative process starts from a set of initial data structures and query plans. We describe how these are created by example. Suppose that we have a sequence $S e q_{U \rightarrow P}$ that consists of a read query on the Users table, followed by the join operation on the Products table. The generator will first create a document candidate for each read query in this sequence, and this candidate data structure contains only the fields that are effectively used. We thus get the data structures $D_{U_{1}}$ and $D_{P_{1}}$, which may be a subset of the original user table $T_{U}$ and product table $T_{P}$. The generator then formulates for each sequence the possible query plans to answer the sequence, using these root data structures. This will yield in this case a single query plan $Q P_{U_{1} \rightarrow P_{1}}$.

Typically, the workload consists of more than one read sequence, and multiple initial data structures are created per sequence. Therefore, we could for example end up with 3 variants of the Users table $D_{U_{1}}, D_{U_{2}}, D_{U_{3}}$. In this case, $D_{U_{1}}$ could 
be the entire normalized Users table, whereas $D_{U_{2}}, D_{U_{3}}$ represent subsets with different columns.

After generating all initial data structures, all possible query plans are generated for all the sequences. The read sequence that utilizes $D_{U_{3}}$ can create at least two query plans, in which one case $D_{U_{3}}$ is used, and in the other case the superset $D_{U_{1}}$. Since this can quickly lead to an exceedingly large set of query plans and candidate data structures, the user can force the generator to only make combinations of entire tables, and not subsets of columns from each table.

\subsection{Query Plan Creation}

Each time query plan optimization results in document structures, the generator will check whether these document structures $D$ are novel. When for example $D_{U_{1} \mid P_{1}}$ is a novel data structure, each query plan that uses $D_{U_{1}}$ or $D_{P_{1}}$ is notified of its existence. Each query plan will then check if it can replace each used document $D$ by the new data structure.

Replacement rules. Suppose the query plan $Q P_{U_{1} \rightarrow P_{1} \rightarrow C_{1}}$ is notified of the existence of $D_{U_{1} \mid P_{1}}$. This will yield the new query plans $Q P_{U_{1} \mid P_{1} \rightarrow P_{1} \rightarrow C_{1}}$ and potentially also $Q P_{U_{1} \rightarrow U_{1} \mid P_{1} \rightarrow C_{1}}$. The latter QP can only be created when either:

Rule 1: The entire set of products is fully contained in $D_{U_{1} \mid P_{1}}$. This is referred to as a fully functional closure (FFC) in literature [17].

Rule 2: The result required to reach $P_{1}$, namely $U_{1}$, was queried in the previous step of the join query, and we are traversing the same relationship.

Query Plan Inner-compaction. These replacements based on the original query plan can typically be compacted. Suppose the query plan $Q P_{U_{1} \mid P_{1} \rightarrow P_{1} \rightarrow C_{1}}$. In this example, we have already obtained the necessary Product information in the first step, and thus this query plan becomes $Q P_{U_{1} \mid P_{1} \rightarrow C_{1}}$. We list the different cases in which such compactions are allowed below:

Case 1: $A \mid B \rightarrow B$ The subsequent data has already been queried.

Case 2: $A \rightarrow A \mid B$ The previous data can also be queried in the next query.

Case 3: $B \rightarrow A|B| C$ If $B$ is a FFC in $A|B| C$, then $B$ can be eliminated.

The query plan can compact itself in multiple iterations until nothing changes.

Secondary Indexing. Suppose we have the Sequence $S e q_{P \rightarrow C}$ that is answered using the query plan $Q P_{U_{1} \mid P_{1} \rightarrow C_{1}}$ which joins Product information with buyer's Comments. Since the Product information is at the $2 n d$-level in the nested document $D_{U_{1} \mid P_{1}}$, the query will require secondary indexing on the nested field Users.Products.id to select the relevant records. Query plans automatically keep track whether they require secondary indexes, which is in turn reflected in their cost. In the schema recommendation, the best query plans will be listed, and when these plans use secondary indexing, it will have to be implemented, which brings an additional cost. 


\subsection{Document Merge Operation}

The merging of data structures is the key underpinning functionality that drives the document generation process. We explain the process by example: suppose we optimize the root query plan $Q P_{U \rightarrow P \rightarrow C}$, then it will attempt to merge together $D_{U}+D_{P}$ and $D_{P}+D_{C}$. When this is allowed, new document structures $D_{U \mid P}$ and $D_{P \mid C}$ are created, which embed relational data into the parent document.

Merge Rules. The merge between two documents $D_{1}$ and $D_{2}$ happens at the initiative of a query plan, and is only allowed when the following rules uphold:

Rule 1: $D_{1} \not \subset D_{2}$. The first document is not a subset of the second.

Rule 2: $D_{1}$.canMerge $\left(D_{2}\right)$. Documents are complex trees of embedding, and their compatibility needs to be checked. The canMerge function checks: (i) if $D_{2}$ answers a subset of the sequences of $D_{1}$, and if either (ii.a) there is an overlap between $D_{1}$ and $D_{2}$, or (ii.b) $D_{1}$ can be connected to the top or bottom of $D_{2}$ its tree.

Rule 3: Let $T=$ merge $\left(D_{1}, D_{2}\right)$, then $\operatorname{Dim}(T) \leq \operatorname{Max} D i m$. The new document's maximum height and width (at each node) must be below the configured thresholds. This prevents the creation of exceedingly large documents.

Rule 4: Let $T=$ merge $\left(D_{1}, D_{2}\right)$, then T.isValidCyclic(). If $\mathrm{T}$ has repeating Entities, then these must correspond with at least one Sequence that makes use of this repetition.

Rule 5: Let $T=$ merge $\left(D_{1}, D_{2}\right)$, then $D_{1} \subset T$ and $D_{2} \subset T$.

Merge between Document Trees. When $D_{1}$ can be merged with $D_{2}$, they are merged at the basis of their entire intersection (the largest overlap) that can be found in the largest tree. If the intersection is empty, the two documents are merged at the first leaf node that can form a connection between the top node of the other tree, or vice versa. This is illustrated in Figure 4.

Merge

(i) Case: overlap

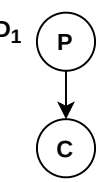

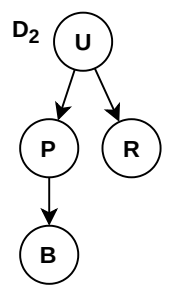

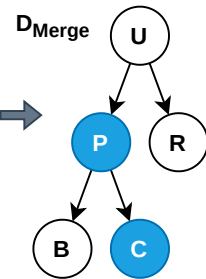

(ii) Case: connection

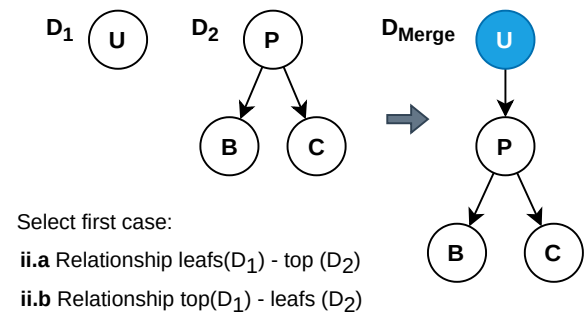

Fig. 4. Document merges at largest overlap or a relationship between two nodes.

The next section explains the mechanism of pruning documents and query plans at the basis of cost models and utility functions. 


\section{Pruning Algorithms}

The set of query plans as generated in the previous steps will be established through many iterations and thus can grow exceedingly large. Since database recommendation is considered to be a NP-hard problem, Section 4.1 describes the mechanism for query plan pruning to force convergence or termination. Once all query plan optimization activities have terminated, we are left with numerous document data structures from which have to select the most suitable. Section 4.2 explains the pruning algorithms employed for this.

\subsection{Query Plan Pruning and Compaction}

At a configurable threshold of query plans (e.g. $\geq 30.000$ ), we can apply two alternative mechanisms to reduce the query plan set: (i) query plan outercompaction, or (ii) query plan pruning.

Query Plan Outer-compaction. In the overall set, a smaller query plan, for example $Q P_{U_{1} \mid P_{1}}$, may be a subset of larger plans such as $Q P_{U_{1} \mid P_{1} \rightarrow P_{1}}$. Therefore, all query plans that are in bucket $k$, and thus have $k$ join steps, are passed to the query plans in bucket $k+1$, which check whether or not they are redundant.

Query Plan Pruning and Cost Models. The second, and main mechanism, maintains an intermediate ranking of all the query plans per Sequence based on their efficiency. The efficiency of a query plan is calculated at the basis of their cost to execute, which takes into account per individual query: (i) the records selected, and (ii) the result size retrieved, which both may be impacted by (iii) the use of secondary indexing.

Query Frequency An important cost factor is the frequency of records selected at a join step, and thus at an individual query. This typically follows the cardinality of the relationship between the first query at this step, and the last query executed in the previous join step. For example, $Q P_{U \rightarrow P}$ first queries a single User record, and subsequently queries an average number of $N_{P}$ Products records owned by the user $U$. However, the query frequency does not always follow the relationship cardinality. For example, query plan $Q P_{U \rightarrow U \mid P}$ also queries a single Users records, but it is only followed by a single Users $/$ Products records. This is because a Users $\mid$ Products record already contains the number of $N_{P}$ records.

Based on their calculable cost, the query plans are sorted and the most costly plans are pruned. We use the ranking of query plans to subsequently rank traversed document structures.

\subsection{Data Structure Pruning}

In the resulting recommendation set, only the $N$ best document structures are chosen along with advice on the ideal query plans to answer the given workload.

The effectiveness of a document $D$ can be defined by the number of total query plans it appears in and their respective query ranks. However, this indicator would simply be skewed by the quantity of plans in which the document 
appears, rather than their quality. Consequentially, we need a metric to determine a query plan's utility, before we can determine the utility of a document.

Query Plan Utility Function. A fitness function for a query plan takes as input its relative rank (with higher being better) between other query plans of the intended read Sequence, and the total rank. The linear fitness function skews the document utility by the quantity of query plans it appears in. The non-linear fitness function distributes more utility to higher-tier query plans (e.g. steps $k=1$ ). We have implemented both a linear, and a non-linear fitness function.

$$
\begin{gathered}
\text { linearFitness }(q p, \text { totalSeqRank })=\frac{2 * q p \cdot \text { Rank }}{\text { totalSeqRank }} \\
\text { nonLinearFitness }(q p, \text { totSeqRank })=\frac{\text { linearFitness }(q p, \text { totSeqRank })}{q p \cdot q u e r y \text { Length }{ }^{2}}
\end{gathered}
$$

Document Ranking. The fitness of a document for a specific query Sequence, is the weighted sum of its relative fitness from its query plans that are subscribed to the document. Each value is also weighted by the query's frequency in the workload to stress its importance. Based on the overall fitness outcomes of each document, they can be ranked from least to most effective.

\section{Evaluation}

We evaluate the DBSR framework in the specific case of the online auction data model and workload found in the RUBiS benchmark [6]. Our evaluation approach is two-fold: (i) we analyse the impact of the configuration parameters on the document generation and pruning process, and (ii) we perform an experimental performance evaluation to assess the quality of recommended schemas and its query plans in a MongoDB cluster, compared to a normalized approach.

\subsection{Document Generation and Pruning}

The parameters that influence the resulting schema and its dimensions the most are: (a) the maximum document tree's height $H$, and (b) the maximum width $W$ which is the maximum number of relationships embedded per single node in the tree, and potentially (c) the degree of pruning.

Table 1 shows the candidate documents in the final document pruning stages, after execution with a maximum document height and node width of respectively $(3,1)$ and $(4,3)$. We ran both configurations with a maximum of 4.000 iterations and pruning threshold of 30.000 query plans. Both executions halt after reaching the maximum number of iterations, and output candidate sets of respectively 26 and 36 documents. Among the overall set of document candidates, we observed a variety of nesting and width, within the specified dimensions of each execution. 
Table 1. Document ranking (low $\rightarrow$ high) during final pruning steps, with $\mathbf{X}$ signifying a removed document. The last step's final document ranks are underlined.

\begin{tabular}{|c|c|c|c|}
\hline \multicolumn{2}{|c|}{ (a) $\operatorname{Max} \operatorname{Dim}(\mathbf{H}=3, \mathbf{W}=1)$} & \multicolumn{2}{|l|}{ (b) $\operatorname{Max} \operatorname{Dim}(\mathrm{H}=4, \mathrm{~W}=3)$} \\
\hline Execution time & $142 \mathrm{~s}$ & Execution time & $166 \mathrm{~s}$ \\
\hline Result size & 26 & Result size & 36 \\
\hline Documents & Rank & Documents & Rank \\
\hline Products $\mid$ Bids & $134 \mathbf{X}$ & Products $\mid$ Comments & $\begin{array}{llllll}1 & 1 & 1 & 1 & 1 & 2\end{array}$ \\
\hline Bids $\mid$ Products & $\begin{array}{lllll}2 & 2 & 3 & 1 & 2\end{array}$ & Products $\mid$ Bids & $255 \mathrm{X}$ \\
\hline Products $\mid$ Users & $\begin{array}{llll}34 & 2 & 3 & \bar{X}\end{array}$ & Products $\mid$ Bids $\mid$ Users & $\begin{array}{lllll}32 & 2 & 2 & 2 & \mathbf{X}\end{array}$ \\
\hline Users & $45 \mathrm{X}$ & Products $\mid$ Bids $\mid$ Users $\mid$ Regions & 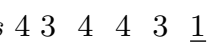 \\
\hline Products $\mid$ Comments & $\begin{array}{lllll}5 & 1 & 1 & 2 & 3\end{array}$ & Users $\mid$ Bids & $5433 \mathrm{X}^{-}$ \\
\hline Products $\mid$ Users $\mid$ Region & 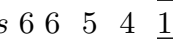 & Products $\mid$ Users & $66 \mathrm{X}$ \\
\hline Comments & $7 \mathrm{X}$ & Comments & $7 \mathbf{X}$ \\
\hline
\end{tabular}

In the final pruning steps and when comparing the different run configurations, both (a) and (b) lead to similar documents, and only documents of width 1 are remaining, though with run (b) having a document of height 4 . In the final step (b) also prunes to a smaller minimum set of 2 documents rather than 3 .

Whenever a document is pruned, for example in (a) when Comments is pruned, the rank of each document will be re-calculated. Subsequently, in that example, Products / Comments is the only document now containing Comments information and consequently, its rank shifts from 5 to 1 . Similar events can be observed throughout the pruning process.

The pruner also skips essential documents when they are the only remaining option to answer a specific query. For example, Regions is only embedded in one document, and is essential to answer a query on a User's Region.

Furthermore, altering the degree of pruning thresholds from a maximum query plan size of $30 \mathrm{~K}$ to $80 \mathrm{~K}$ only results in a minor change in (b), and yields Bids|Products over Products|Bids.

\subsection{Performance Evaluation}

We evaluate the quality of DBSR's schema recommendations in the document database MongoDB, selected for its widespread popularity and maturity.

The evaluation compares two schema recommendations from Table 1 (a), which feature respectively 3 and 5 document collections, to a normalized schema consisting of the 5 original tables (as shown in Figure 1). The recommender's input workload is executed against these schemas and consists of 11 JOIN queries.

Evaluation setup. In order to conduct the evaluation, we extended the YCSB NoSQL benchmark [9] with a custom data loader and workload model based on the online auction case (RUBiS benchmark [6] $)^{2}$. The MongoDB database is a

\footnotetext{
${ }^{2}$ Benchmark repository: https://github.com/vreniers/YCSB-MongoDB-RUBiS
} 
3-node cluster on Amazon AWS; the primary node serves as a query router, and two shards horizontally partition the data based on the primary key. Secondary indexing is implemented per document collection when required by a query plan.

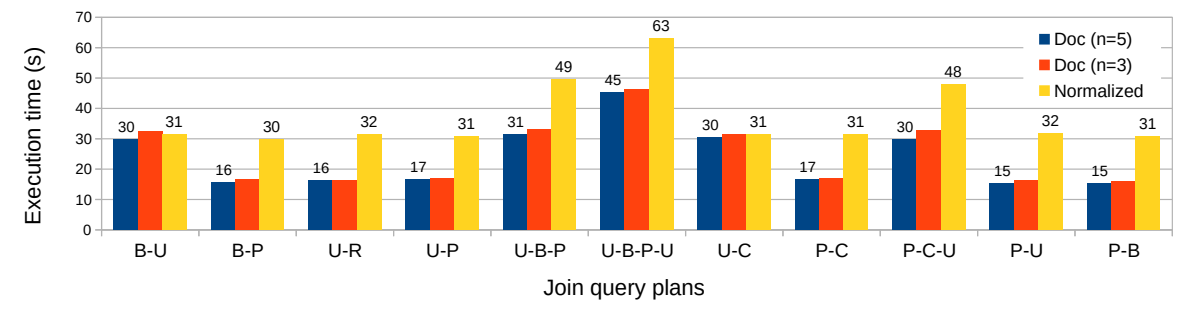

Fig. 5. Query join performance on recommended and normalized schemas.

Results. Figure 5 shows the execution time per join query, executed numerously, on three different schemas. For example, $Q P_{P \rightarrow B}$ queries a Product's information and all its Bids. The recommended schemas in most cases outperform the normalized schema, and in other cases, the baseline performance is similar. These performance gains stem from eliminating costly JOIN queries, in which the intermediate results are returned to the client at each step to formulate the follow-up query. Despite using only 3 document collections, many queries can be answered with a single look-up. In terms of the increase in data redundancy when compared to the normalized schema, mainly Product information is duplicated three times, and User with Region information in the document set $D_{P|U| R}$.

Using a slightly larger set of 5 documents yielded no discernible difference over using the minimal schema recommendation. This can be attributed to the manner in which the documents are ranked, namely they are sorted based on their global utility to all queries. It could be the case that a single document is highly effective for a single or few queries, but not globally. To take such cases into account, the relative weight of these queries can be increased which ranks their utilized documents higher, or by implementing a more local optima search.

\section{Related Work}

Table 2 shows an overview of all other existing schema tools for NoSQL databases, and we distinguish between (i) transformation tools that essentially apply transformation rules (e.g. heuristics) to a data model to result in a target schema, and (ii) generators, which enumerate multiple data structures.

Many tools shown in Table 2 simply apply a straightforward conversion from for example an ER-model guided by queries (e.g. Chebotko et al. [7]) and yield a somewhat optimal schema. While C. Lee et al. [15] enumerate all possible columnar structures by enumerating over foreign dependencies, it lacks a cost model and therefore does not provide a schema recommendation. 
Table 2. Overview of schema conversion tools and recommenders.

\begin{tabular}{|c|c|c|c|c|c|c|c|}
\hline Transformation & Workload & Schema & Dupli. & Nest & Cost model & Index & Plans \\
\hline Chebotko et al.[7] & Read $(\mathrm{J}, \mathrm{O}, \mathrm{E})$ & Columnar & Yes & Yes* & No & $\mathrm{C}$ & No \\
\hline Mortadelo [22] & $\operatorname{Read}(\mathrm{J}, \mathrm{O}, \mathrm{E})$ & $\mathrm{Col} / \mathrm{Doc}$ & Yes & Yes & No & $\mathrm{C}$ & No \\
\hline De Lima et al.[17] & Frequency (R) & Document & No & Yes & No & - & No \\
\hline Jia et al. [13] & Freq $(\mathrm{W} / \mathrm{R}(\mathrm{J}))$ & Document & Yes & Yes & Table size & - & No \\
\hline Zhao et al. [23] & - & Nested & Yes & Yes & No & - & No \\
\hline QODM $[16]$ & Read $(\mathrm{J})$ & Generic & Yes & Yes & No & - & No \\
\hline Banerjee et al. [4] & - & JSON & No & Yes & No & - & No \\
\hline \multicolumn{8}{|c|}{ Schema Generators } \\
\hline Lee et al. [15] & - & Columnar & Yes & Yes* & No & - & No \\
\hline Bermbach et al. [5] & $\mathrm{R}(\mathrm{J}, \mathrm{E}), \mathrm{W}$ & Columnar & Yes & Yes* & Schema rank & $\mathrm{S}$ & No \\
\hline NoSE [18] & $\mathrm{R}(\mathrm{J}, \mathrm{O}, \mathrm{E}), \mathrm{W}$ & Columnar & Yes & Yes* & Query cost & $\mathrm{C}$ & Yes \\
\hline DBSR & Read $(\mathrm{J})$ & Document & Yes & Yes & Extensive & $\mathrm{S}$ & Yes \\
\hline
\end{tabular}

Abbreviations: Read (R), Write (W), Join (J), Ordering (O), Equality search (E), Clustering index $(\mathrm{C})$, Secondary index $(\mathrm{S}),{ }^{*}$ Groups data together.

\subsection{In-depth Comparison}

We provide an in-depth comparison between DBSR and the approaches of schema generators by Bermbach et al. [5] and Mior et al. (NoSE) [18], which provide schema recommenders for NoSQL columnar stores (e.g. Cassandra). Despite working on fundamentally different data structures, they follow a similar approach in enumerating data structures and cost-based elimination.

Candidate Generation. The initial process in Bermbach et al. [5] loops over each JOIN query and creates a single materialized view table, enabling a single lookup per query. NoSE [18] creates materialized views for each step in the JOIN query similar to our approach. In Bermbach et al. [5], multiple schemas are calculated as the cartesian product of singular materialized views. In each potential schema, it is then evaluated whether a data structure can be used for another query. In NoSE [18] candidate combinations are merged when they meet certain requirements: identical primary key, no clustering or secondary indexes, and have a different set of data attributes. In our approach, we can flexibly merge data structure candidates, at various points (e.g. overlap, or connection), and only when JOIN queries benefit from such a merge. In a second stage, NoSE [18] and Bermbach et al. [5] can add data structures (i.e. look-up tables) when required by write/update queries. A similar approach could also be implemented in DBSR.

Schema Assessment. In both approaches, every potential schema option is created as every possible combination of data structures that constitute a valid schema. In contrast, we calculate the optimal schema by pruning the least effective document structures using a non-linear utility heuristic. In Bermbach et al. [5], each schema is ranked in terms of a simple scoring function that builds on two metrics: (i) average number of secondary indexes and (ii) average amount 
of data duplication. In NoSE [18], a query cost model is implemented, and each schema is assessed by the total cost of executing all queries against this schema.

\section{Conclusions}

In this paper, we have presented a document-based schema recommender (DBSR) for NoSQL document-oriented databases. The DBSR framework provides several contributions as re-usable modules, namely (i) a workload-driven document generator, with (ii) cost-based pruning algorithms for query plans and document data structures. These modules, combined in the framework, can provide a schema recommendation for an ER data model and read workload. The schema recommendation features document structures, query plans and secondary indexing suggestions, along with a utility matrix that provides insights into the efficiency of involved documents and query costs.

Our evaluation provides (i) an analysis of the schema generation and pruning process, and (ii) an experimental performance evaluation of a recommended schema using an extension of YCSB in MongoDB. Significant performance benefits were shown for read queries, at the expense of data redundancy. The pruning approach eliminates documents based on their relative global utility.

In future work, we would like to investigate alternative pruning-based approaches (e.g. more local solution space exploration), as well as NoSQL benchmarks that evaluate NoSQL schema recommendations that function on ER models, rather than key-value pairs in YCSB. The further integration between databases, (micro-)benchmarking, and schema recommendation will allow evalu-

ating candidates automatically, and subsequently calibration of the cost models used tailored to the selected use case, database technology and deployment.

\section{Acknowledgments}

This work has been funded by the KU Leuven Research Fund.

\section{References}

1. Entity relationships and document design. https://docs.couchbase.com/server/4.6/datamodeling/entity-relationship-doc-design.html, accessed: 2020-05-25

2. MongoDB: Data model design. https://docs.mongodb.com/manual/core/datamodel-design/, accessed: 2020-05-25

3. Atzeni, P., Bugiotti, F., Cabibbo, L., Torlone, R.: Data modeling in the NoSQL world. Computer Standards \& Interfaces 67, 103149 (2020)

4. Banerjee, S., Sarkar, A.: Logical level design of nosql databases. In: 2016 IEEE Region 10 Conference (TENCON). pp. 2360-2365 (2016)

5. Bermbach, D., Müller, S., Eberhardt, J., Tai, S.: Informed schema design for column store-based database services. In: 2015 IEEE 8th International Conference on Service-Oriented Computing and Applications (SOCA). pp. 163-172 (Oct 2015)

6. Cecchet, E., Marguerite, J., Zwaenepoel, W.: Performance and scalability of EJB applications. ACM Sigplan Notices 37(11), 246-261 (2002) 
7. Chebotko, A., Kashlev, A., Lu, S.: A Big Data Modeling Methodology for Apache Cassandra. In: IEEE International Congress on Big Data (2015)

8. Chun-Hung Cheng, Wing-Kin Lee, Kam-Fai Wong: A genetic algorithm-based clustering approach for database partitioning. IEEE Transactions on Systems, Man, and Cybernetics, Part C (Applications and Reviews) 32(3), 215-230 (2002)

9. Cooper, B.F., Silberstein, A., Tam, E., Ramakrishnan, R., Sears, R.: Benchmarking cloud serving systems with YCSB. In: Proceedings of the 1st ACM symposium on Cloud computing. pp. 143-154 (2010)

10. Gómez, P., Casallas, R., Roncancio, C.: Data schema does matter, even in NoSQL systems! In: IEEE Tenth International Conference on Research Challenges in Information Science (RCIS) (2016)

11. Gómez, P., Roncancio, C., Casallas, R.: Towards quality analysis for document oriented bases. In: International Conference on Conceptual Modeling (2018)

12. Grolinger, K., Higashino, W.A., Tiwari, A., Capretz, M.A.M.: Data management in cloud environments: NoSQL and NewSQL data stores. Journal of Cloud Computing: Advances, Systems and Applications 2(1), 22 (2013)

13. Jia, T., Zhao, X., Wang, Z., Gong, D., Ding, G.: Model Transformation and Data Migration from Relational Database to MongoDB. In: IEEE International Congress on Big Data (BigData Congress) (2016)

14. Kanade, A., Gopal, A., Kanade, S.: A study of normalization and embedding in MongoDB. In: IEEE International Advance Computing Conference (IACC) (2014)

15. Lee, C., Zheng, Y.: Automatic SQL-to-NoSQL schema transformation over the MySQL and HBase databases. In: 2015 IEEE International Conference on Consumer Electronics - Taiwan (2015)

16. Li, X., Ma, Z., Chen, H.: QODM: A query-oriented data modeling approach for NoSQL databases. In: 2014 IEEE Workshop on Advanced Research and Technology in Industry Applications (WARTIA). pp. 338-345. IEEE (2014)

17. de Lima, C., dos Santos Mello, R.: A workload-driven logical design approach for NoSQL document databases. In: Proceedings of the 17th International Conference on Information Integration and Web-based Applications \& Services (2015)

18. Mior, M.J., Salem, K., Aboulnaga, A., Liu, R.: NoSE: Schema Design for NoSQL Applications. IEEE Transactions on Knowledge and Data Engineering (Oct 2017)

19. Pasqualin, D., Souza, G., Buratti, E.L., de Almeida, E.C., Del Fabro, M.D., Weingaertner, D.: A case study of the aggregation query model in read-mostly NoSQL document stores. In: Proceedings of the 20th International Database Engineering \& Applications Symposium (2016)

20. Reniers, V., Van Landuyt, D., Rafique, A., Joosen, W.: Schema design support for semi-structured data: Finding the sweet spot between NF and De-NF. In: 2017 IEEE International Conference on Big Data (Big Data). pp. 2921-2930 (2017)

21. Stonebraker, M.: SQL Databases v. NoSQL Databases. Commun. ACM 53(4), 1011 (Apr 2010). https://doi.org/10.1145/1721654.1721659

22. de la Vega, A., García-Saiz, D., Blanco, C., Zorrilla, M., Sánchez, P.: Mortadelo: Automatic generation of NoSQL stores from platform-independent data models. Future Generation Computer Systems 105, 455-474 (2020)

23. Zhao, G., Lin, Q., Li, L., Li, Z.: Schema conversion model of SQL database to NoSQL. In: 2014 Ninth International Conference on P2P, Parallel, Grid, Cloud and Internet Computing. pp. 355-362 (Nov 2014) 